\title{
Fairness Analysis in Cellular Networks using Stochastic Petri Nets
}

\author{
Rainer Schoenen*, Akram Bin Sediq*, Halim Yanikomeroglu*, Gamini Senarath ${ }^{\S}$, and Zhijun Chao ${ }^{\S}$ \\ * Department of Systems and Computer Engineering, Carleton University, Ottawa, Ontario, Canada \\ $\S$ Huawei Technologies Canada Co., LTD.
}

\begin{abstract}
Cellular wireless networks based on OFDMA utilize adaptive modulation and coding to operate effectively in regions of high and low SINR. Therefore the local single antenna spectral efficiency ranges between 5 in the cell center and $0.2 \mathrm{~b} / \mathrm{s} / \mathrm{Hz}$ at the cell edge. The scheduling goal of high average spectral efficiency contradicts the goal of a good rate fairness among all terminals. Also there is a tradeoff between increasing the cell edge performance and maintaining a high average spectral efficiency. In this paper a stochastic Petri net analysis approach is taken and a numeric analysis is performed based on Markov chain equivalence and steady state calculations. The proposed models are deliberately abstract but offer commonly used tuning parameters in order to study the tradeoff without too many degrees of freedom.
\end{abstract}

Index Terms-stochastic Petri nets; fairness; user in the loop

\section{INTRODUCTION}

W IRELESS cellular networks feature adaptive modulation and coding (AMC) on all subchannels of OFDMA, so the variation of SINR translates into a variation of local spectral efficiency $\gamma$ (mutual information MI) in the range of 0.2 to $5 \mathrm{~b} / \mathrm{s} / \mathrm{Hz}$ from the cell edge to the center. Figure 1 shows the typical AMC modes of LTE-Advanced and their performance, upper bounded by the Shannon capacity. The same ordering is used in Figure 2. It shows the probability of each AMC mode in the cellular scenario Urban Macro (UMa) defined by IMT-Advanced evaluation guidelines and has been obtained using numeric analysis [1]. They are independent from the number of user terminals (UT) in the cell.

Resource scheduling in a cell can be performed with different goals. The highest spectral efficiency averaged over the whole cell area $(\bar{\gamma})$ is achieved by the Max-SINR (MS) scheduler, on the expense of cell edge performance $\left(\gamma_{C E}\right)$. Other schedulers like proportional fair (PF) and round robin (RR) can trade off between increasing the cell edge performance and maintaining a high average spectral efficiency.

While traditional evaluation methods require simulation and detailed scheduler implementations, this paper presents an abstract simulation-free model based on stochastic Petri nets (SPN). The numeric analysis is based on the Markov chain equivalence of SPNs and steady state probabilities are calculated to obtain reward measures. The proposed models offer commonly used tuning parameters to evaluate the tradeoff between $\bar{\gamma}, \gamma_{C E}$ and fairness $J$. This paper introduces the SPN model, defines scheduling parameters and obtains parameter analysis results. Finally, a User-in-the-loop [2] based UT relocation mechanism is analysed.
In need for a theoretic system model to capture physical layer effects abstract enough for higher layers, Petri nets [3] are an abstract way for modeling Markov chains (MC) with arbitrary connectivity but maintain a clearly arranged graphical structure. Their advantage is the combination of the flexibility of a Turing-complete automaton with the power of stochastic MC analysis. SPN and generalized SPN (GSPN) [4] have become a useful tool for adept researches in computer science. Results are obtained by numeric tools and do not require simulation. Useful tools for GSPN analysis exist [5]. In recent years, GSPN have been used occasionally to model communications systems [6], [7] and protocols [8], but a widespread use is not observed, mainly due to fact that SPN are unknown to most wireless researchers, unaware that Markov chains are a subset of SPN.

A number of promising SPN approaches already exist in all fields of wireless communications, e.g., resource management in cellular wireless systems [9], multihop (relay) transmissions [10] and IEEE 802.16 [11]. Links to higher layers exist in works for TCP models [12] or Credit-Based Flow Control [13]. Wireless Channel Models can be found in [10].

There is a demand for modeling technologies like LTE-A and other IMT-Advanced systems [14]. Especially ISO/OSI layer two (medium access control) can benefit from GSPN analysis methods. The importance of packet delay aware models for resource and packet scheduling [15] and radio resource management [16] is obvious. Most works study the maximum throughput with a full buffer assumption, because their methodology is unable to model traffic. Therefore these studies assume the overload condition and cannot provide finite delay results.

The paper is organized as follows: Section II introduces the basics of stochastic Petri nets, followed by the section III on the SPN model of the radio cell with fairness versus rate objective. Performance results are presented in section IV.

\section{Petri Nets}

Petri nets (PN) is a graphical and mathematical tool suitable to model complex systems with a state. Systems can be be described and studied when they are concurrent, asynchronous, distributed, deterministic or stochastic. Subclasses of PN are finite state machines and marked graphs used for DSP algorithms [17]. Many aspects of flow charts and description languages can be modeled with PN. There is plenty of literature on the underlying graph theory, liveness analysis, 


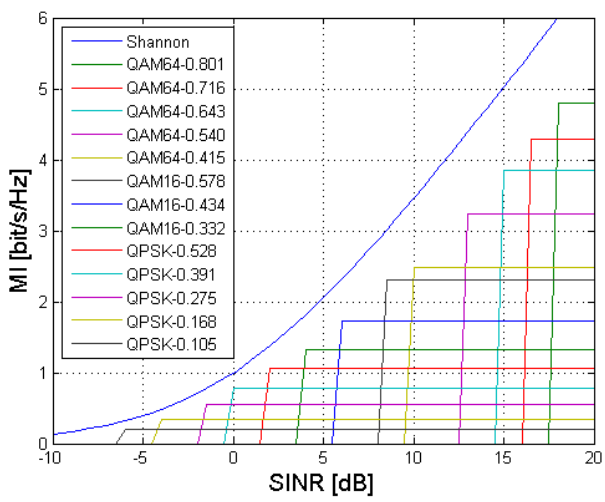

Fig. 1. Adaptive Modulation and Coding (AMC) performance of LTE-A

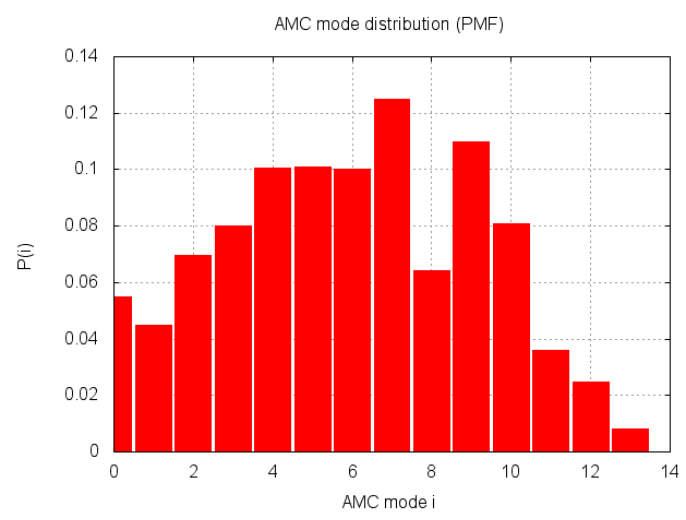

Fig. 2. LTE-A AMC probability $\pi_{i}$ in unconstrained UMa scenario

reachability set and other properties [3]. A PN is defined as a directed, weighted, bipartite graph having two sets of nodes called places $\left(P_{i}\right)$ and transitions $\left(T_{j}\right)$. Places are drawn as circles, transitions as boxes. Input arcs connect certain $P_{i}$ to $T_{j}$ with weight $w_{i j}$, output arcs connect $T_{j}$ to $P_{i}$ with multiplicity $v_{i j}$. Together they form the incidence matrix $\mathbf{D}=\left[v_{i j}-w_{i j}\right]$ When a PN consists of $M$ transitions and $N$ places, $\mathbf{D}$ is a $M \times N$ matrix (m rows, $\mathrm{n}$ columns). Places can contain an integer number of tokens (dots), all of which constitute the state called marking $\vec{m}$. The initial marking $\vec{m}_{0}$ is the start state. The notation $\# P_{i}=m_{i}$ means the current number of tokens in place $P_{i}$. An atomic action is the firing of a transition $T_{j}$ which changes the marking to

$$
\vec{m}_{k}=\vec{m}_{k-1}+\vec{t}_{k-1} \cdot \mathbf{D}
$$

with the firing vector $\vec{t}_{k-1}$ which is all zero except a single ' 1 ' at the j.th index. For further details of firing rules, disabling arcs, liveness and reachability see [3].

\section{A. Stochastic Petri Nets}

Stochastic PN (SPN) extend the paradigm to model time, essentially by assigning each transition a firing rate (in the continuous time case (CT)) or a firing probability (in the discrete time case (DT)). Here we will focus on the CT case only. Then the firing rates are given by $\vec{\Lambda}=\lambda_{1}, \ldots, \lambda_{m}$ which can be marking-dependent. $\lambda_{j}$ is the inverse of the average firing time $t_{j}$. Firing times are exponentially distributed, therefore memoryless, and the resulting reachability graph (RG) forms a Markov chain (MC) [4]. All arcs in the RG are annotated with the $\lambda_{j}$ of the transition $T_{j}$ responsible for the state change. The matrix $\mathbf{R}$ of all arc rates can then be used to calculate the steady-state solution of the MC and from that all other performance metrics can be obtained.

Generalized SPN (GSPN) combine both immediate (thick bar) and timed transitions (empty box), which also reduce to MC after condensing all tangible states, but offer a much higher modeling power. Priorities and weights can easily be annotated to immediate transition to model the outcome likelihood of deterministic and random decisions. Deterministic and stochastic SPN (DSPN) also allow one enabled transition with deterministic (fixed) firing time, denoted with a filled box.

There is decent tool support for SPN and the MC is automatically determined and solved [5].

Queueing models and networks are a subset of the modeling power of SPN, and results for queue length and waiting times can easily be derived [18], that is why SPNs are becoming increasingly popular for modeling communication networks [6].

\section{ABSTRACT SPN MODEL}

The SPNs in Figure 3 represent different schedulers. They have in common that the selection of a UT having a particular average AMC mode is indicated by the token being in one of the places $P 00$ to $P 13$, where $P 00$ represents temporary outage, i.e. no available AMC mode. According to Figure 1 this is a translation from the SINR distribution. In Figure 3(a) the $\mathrm{AMC}(\mathrm{UT})$ selection is modeled by probabilistic weights $w_{i}$ on the transitions $A M C 00$ to $A M C 13$. In an unconstrained operation, the weights are simply defined as $w_{i}=\pi_{i}$, the known occurence from Figure 2. The probabilities $\pi_{i}$ were obtained before by simulation [19] in the UMa IMT-A scenario with realistic conditions, including interference and shadowing. The timing behavior is determined by transition Timer, and the rest of the SPN is constructed to conserve (limit) the token count in a loop, so that the PN is bounded, live and the Markov chain has a limited number of states. This model abstracts from (a limited integer count of) individual users but instead the model corresponds to a continuous user density in the cell area.

Figure 3 (b) adds the round robin ( $R R 1$ to $R R 13$ ) component to it, so that each resource will be scheduled equally likely.

Figure 3(c) models the performance of the User-in-the-loop (UIL) concept [2], where UTs can be redirected to locations of better $\gamma$. Therefore the first selection in places $P \# \# V$ is modified by movements towards better AMC modes. E.g., a token from originally $P 01 \mathrm{~V}$ can move to $P 09$ and end up with a better performance. Transitions between $P 00$ and $P 01$ and so on happen with probability $p$ and the straight transitions $T 00 \mathrm{~V}$ and below fire with $(1-p)$.

The models above represent the full buffer situation (all UTs have packets available to send). SPN additionally allow queueing analysis of systems with individual packets (tokens), 

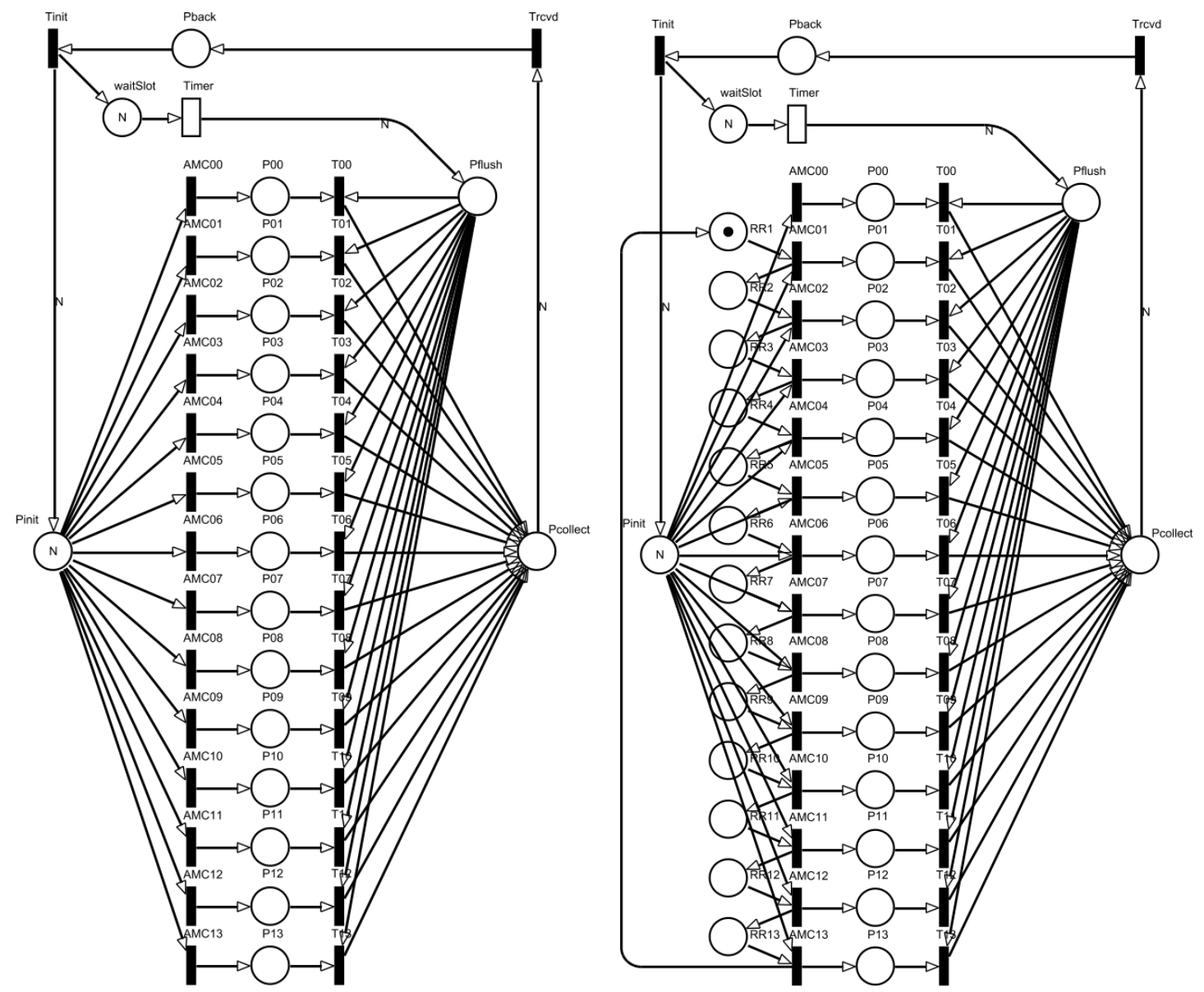

(a) SPN of UT selection with weights $w_{i}$ on transitions $A M C_{i}$

(b) SPN of UT selection with round-robin UT selection

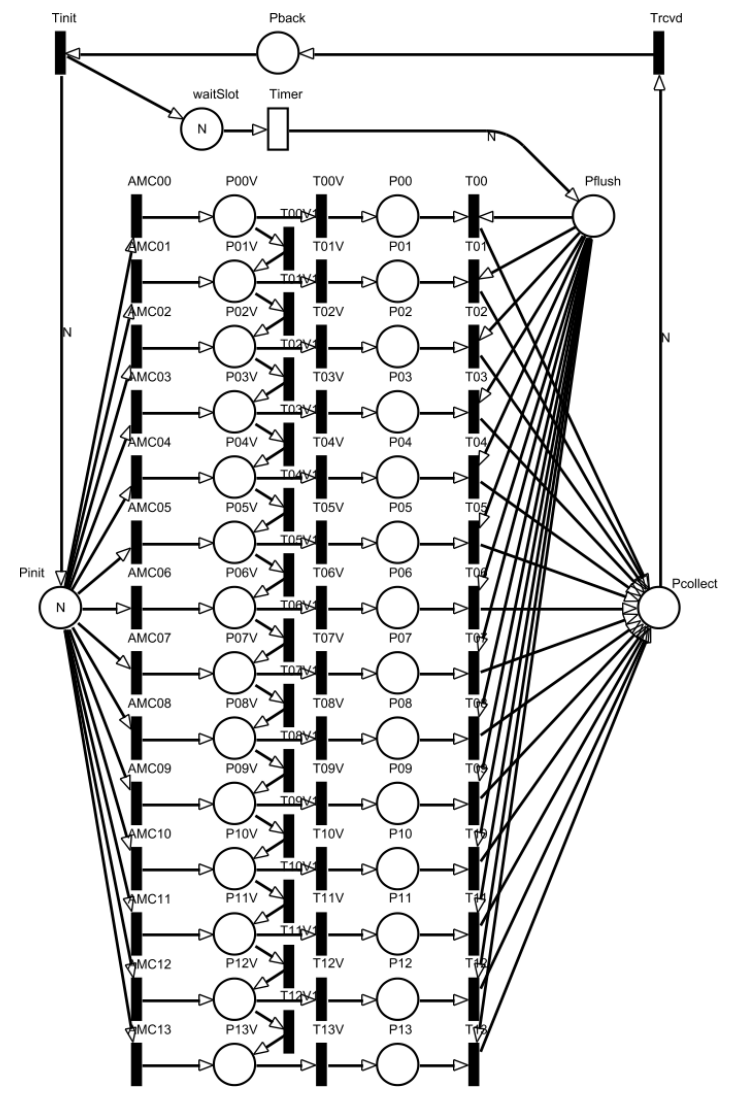

(c) SPN of weighted UT selection and UIL-controlled user

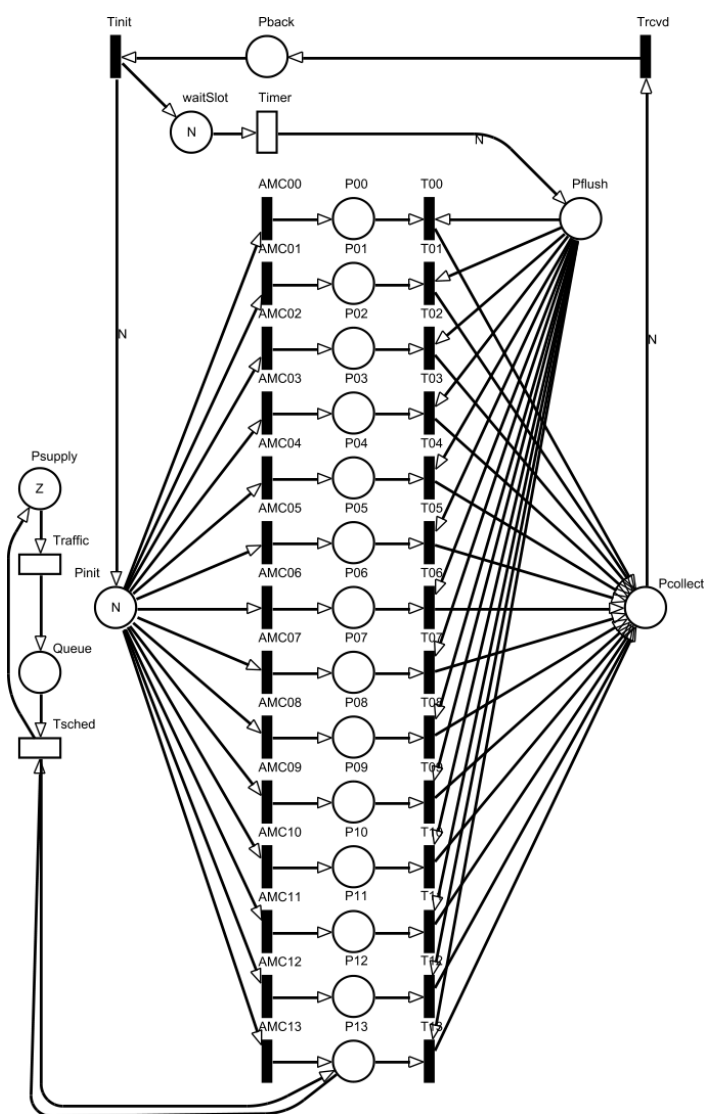

(d) SPN of queueing analysis on one UT movement

Fig. 3. Stochastic Petri Nets of the analyzed UMa scenario with different schedulers. Places P\#\# model the UT selection of the scheduler 
realistic service and a traffic load $\rho<1$. Figure 3(d) shows the extension of an attached queue to the highest AMC mode, representing a UT in the cell center.

Fairness is commonly defined by the Jain's fairness index:

$$
J(\vec{r})=\frac{\left(\sum_{i=1}^{N} r_{i}\right)^{2}}{N \cdot \sum_{i=1}^{N} r_{i}^{2}}
$$

It can be used to evaluate scheduling fairness among different UTs. $J_{R}$ is defined as rate fairness when it compares data rates $r_{i}$, but a resource fairness $J_{S}$ can also be defined when comparing the number of assigned resources $R_{i}$ per UT $i$.

The weights in Figure 3(a) are now adjustable by parameters, so that the different goals can be traded off. In a Proportional Fair scheduler the temporary dynamic priority of a connection $i$ is given by

$$
P_{i}=\frac{\gamma_{i}^{h}}{H_{i}^{\beta}}
$$

where $\gamma_{i}$ is the currently assumed AMC(i) and $H_{i}$ is the historical average rate of UT $i$. The weights $w_{i}$ in the SPN are now defined as:

$$
w_{i}=\pi_{i} \cdot\left(1-s+s \cdot \gamma_{i}^{h}\right)
$$

This allows scaling from unconstrained mode $(s=0)$ to $\gamma_{A M C}$-proportional mode $(s=1)$ and exponential emphasis by parameter $h \in[-2 ; 2]$. Therefore the UT selection can be biased proportional to $\gamma_{A M C}(h=1)$, independent of $\gamma_{A M C}$ $(h=0)$, or inversely proportional to $\gamma_{A M C}(h=-1)$. More extreme emphasis is possible by any real $|h|>1$ or $\propto \sqrt{\gamma}$ by $h=\frac{1}{2}$. $h=1$ gives preference to UTs with high $\gamma$, so $\bar{\gamma}$ is expected to be high on the expense of $\gamma_{C E}, J_{R}$ and $J_{S}$. $h=-1$ gives more resources to cell edge UTs, therefore $\bar{\gamma}$ should go down while $\gamma_{C E}$ is increased. It should be noted that fairness tradeoffs are mainly relevant for best effort (BE) traffic.

\section{PERFormanCE}

Parameterized experiments have been performed with all models on an SPN analysis tool [5]. The reward measures are obtained from the token distribution $p_{i}$ :

$$
\begin{gathered}
\bar{\gamma}=\sum_{i=0}^{13} p_{i} \cdot \gamma_{i}, \quad \gamma_{C E}=\operatorname{Pr}(\# P 01) \cdot \gamma_{01} \\
J_{R}=\frac{\left(\sum_{i=1}^{13} p_{i} \cdot \gamma_{i}\right)^{2}}{13 \cdot \sum_{i=1}^{13}\left(p_{i} \cdot \gamma_{i}\right)^{2}}, \quad J_{S}=\frac{\left(\sum_{i=1}^{13} p_{i}\right)^{2}}{13 \cdot \sum_{i=1}^{13} p_{i}^{2}}
\end{gathered}
$$

The main SPN in Figure 3(a) leads to the results in Figure 4. Without scheduler tuning, the reference values are $\bar{\gamma}=1.58$ $\mathrm{b} / \mathrm{s} / \mathrm{Hz}, \gamma_{C E}=0.0094 \mathrm{~b} / \mathrm{s} / \mathrm{Hz}, J_{R}=0.680$ and $J_{S}=0.818$. The parameter analysis now varies $s$ and $h$ and studies the impact on the two fairness metrics $J_{R}$ and $J_{S}$ and spectral efficiencies $\bar{\gamma}$ and $\gamma_{C E}$.

With full penetration factor $s=1$, a higher parameter $h$ indeed tunes up $\bar{\gamma}$ (Figure 4(a)), whereas negative $h$ are suitable to increase cell edge $\gamma_{C E}$ (Figure 4(c)). The effect of different $s$ is displayed in Figures 4(b), 4(d) and 4(f). The main tradeoff of $\gamma_{C E}$ vs $\bar{\gamma}$ is shown in Figure 6(b) and allows to explore the feasible region from one extreme (all resources to the cell edge) to the other extreme (all resources to the cell center). This can be compared to other results in the literature [20].

Rate fairness goes down with $h$ as in Figure 4(e) but can become maximum with $h=2$ and $s=0.5$ as in Figure 4(f). Its tradeoff is shown in Figure 6(a). We observe that ideal rate fairness cannot be achieved with the given parameters, but even the maximum at $J_{R}=0.8$ already degrades the spectral efficiency to below $1 \mathrm{~b} / \mathrm{s} / \mathrm{Hz}$. Resource fairness will be unbalanced with each change of $h$ or $s$, as shown in Figure 5.

The results of Round Robin in Figure 3(b) are $\gamma=$ $0.8925928, \gamma_{C E}=0.0069424, J_{R}=0.6563011, J_{S}=1.0$, as expected, because it treats each AMC mode equal.

The UIL performance of Figure 3(c) is shown in Figure 7: Without participation $\bar{\gamma}(p=0)=1.58$ stays unchanged, but with more and more users participating, $\bar{\gamma}$ rises monotonously with $p$ up to the theoretical limit of $\gamma_{13}$. Falling values in Figure 7(b) are no reason for worries, because the meaning is here that less UTs will be located in the cell edge after relocation.

\section{CONCLUSION}

This paper addresses the scheduling tradeoff between average cell spectral efficiency, cell edge performance and fairness in cellular networks. The system is abstractly modeled as a stochastic Petri net which incorporates parameters that model real scheduler tuning parameters. Results were obtained by Markov chain steady state analysis, not by simulation. The numbers show that a stepless tradeoff is always possible but a single scheduling goal does not exist. The contributed model is shown to be applicable for (resource) proportional fairness, round robin and even the user-in-the-loop approach.

\section{REFERENCES}

[1] M. Mühleisen, D. Bültmann, and R. Schoenen, "Analytical Validation of an IMT-Advanced Compliant LTE System Level Simulator," in Proceedings of the European Wireless, Vienna, Apr 2011.

[2] R. Schoenen, "On increasing the spectral efficiency more than $100 \%$ by user-in-the-control-loop," in Proc. of the 16th Asia-Pacific Conference on Communications (APCC), Auckland, October 2010.

[3] T. Murata, "Petri Nets: Properties, Analysis and Applications," Proceedings of the IEEE, vol. 77, no. 4, pp. 541-581, April 1989.

[4] M. Marsan, Modelling with Generalized Stochastic Petri Nets. Wiley, 1996, iSBN 0-471-93059-8.

[5] R. German, "A toolkit for evaluating non-markovian stochastic Petri nets," Performance Evaluation, vol. 24, pp. 69-87, 1995.

[6] J. Billington et al., Application of Petri Nets to Communication Networks. Springer, 1999, ISBN 3-540-65870-X.

[7] L. Lei, C. Lin, J. Cai, and X. Shen, "Performancs analysis of wireless opportunistic schedulers using stochastic Petri nets," IEEE Transactions on Wireless Communications, vol. 8, no. 4, April 2009.

[8] M. Bosch and G. Schmid, "Generic petri net models of protocol mechanisms in communication systems," Computer Communications, vol. 14 , no. 3 , pp. 143 - 156, 1991.

[9] F. Jaimes-Romero, D. Munoz-Rodriguez, C. Molina, and H. Tawfik, "Modeling resource management in cellular systems using Petri nets," in IEEE Transactions on Vehicular technology, May 1997, pp. 298-312.

[10] R. Schoenen, M. Salem, A. Sediq, and H. Yanikomeroglu, "Multihop wireless channel models suitable for stochastic Petri nets and markov state analysis," in Proceedings of IEEE VTC Spring, Budapest, May 2011.

[11] S. Geetha and R. Jayaparvathy, "Modeling and Analysis of bandwidth Allocation in IEEE 802.16 MAC: A Stochastic Reward net Approach," Int. J. Communications, Network ans System Sciences, vol. 3, no. 7, pp. 631-637, July 2010. 
RESULTMl $=f(h)$

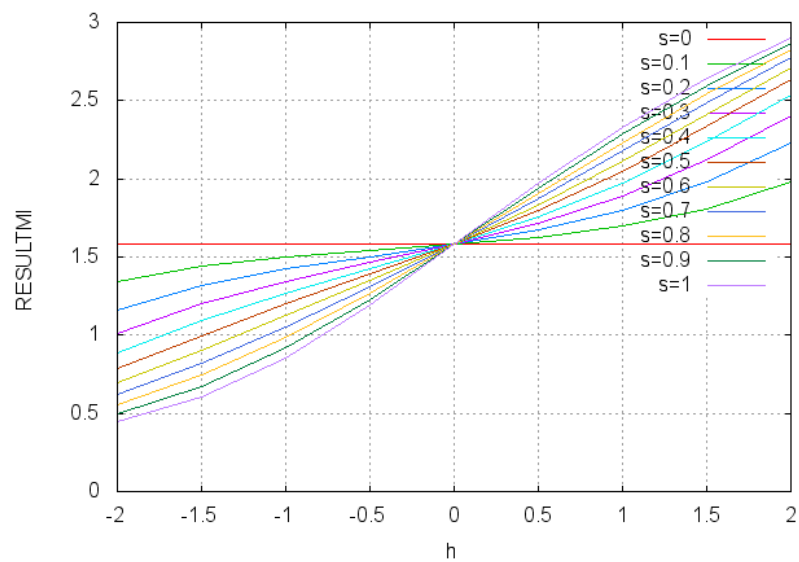

(a) Spectral efficiency $\bar{\gamma}=f(\underline{\mathbf{h}}, s)$ in $\mathrm{b} / \mathrm{s} / \mathrm{Hz}$

CELLEDGEMl $=\mathrm{f}(\mathrm{h})$

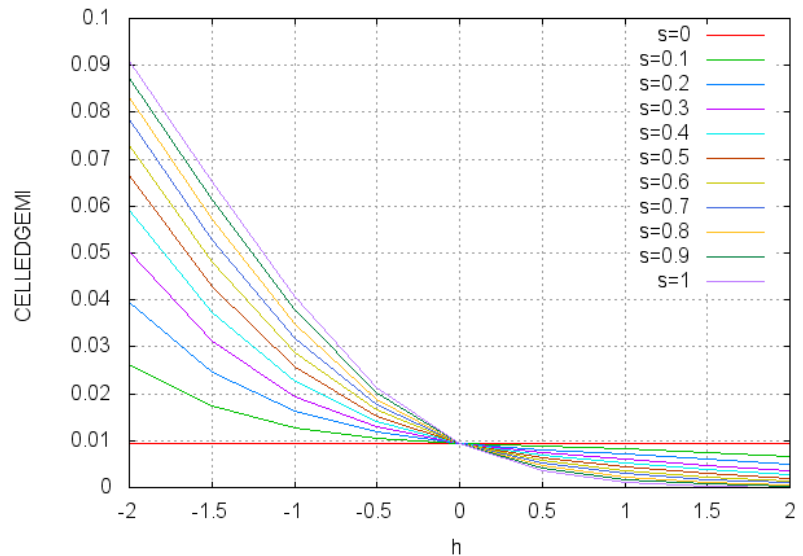

(c) Cell edge performance $\gamma_{C E}=f(\underline{\mathbf{h}}, s)$ in $\mathrm{b} / \mathrm{s} / \mathrm{Hz}$

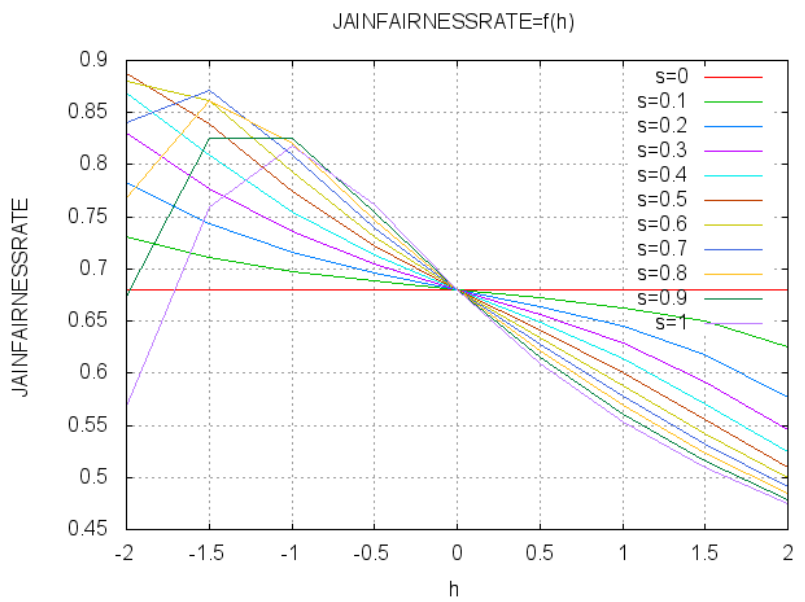

(e) Rate fairness $J_{R}=f(\underline{\mathbf{h}}, s)$
RESULTMI =f(s)

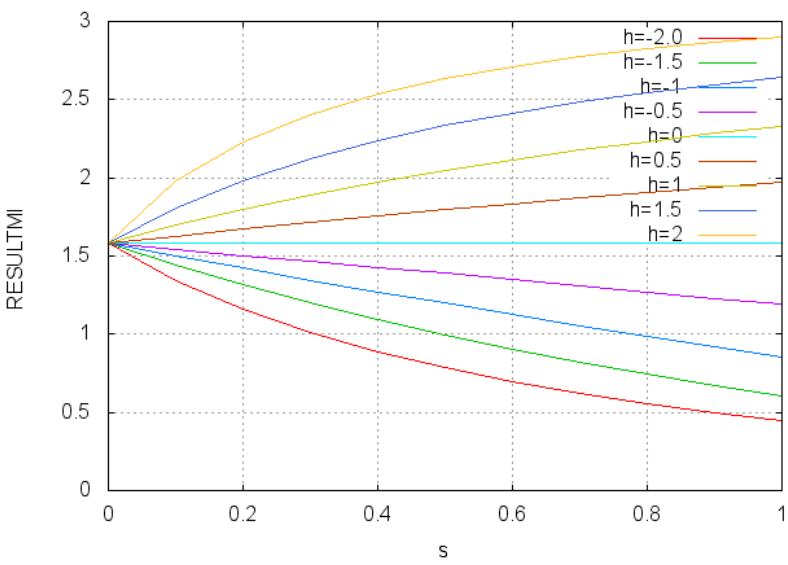

(b) Spectral efficiency $\bar{\gamma}=f(h, \underline{\mathbf{s}})$ in $\mathrm{b} / \mathrm{s} / \mathrm{Hz}$

CELLEDGEMI $=f(s)$

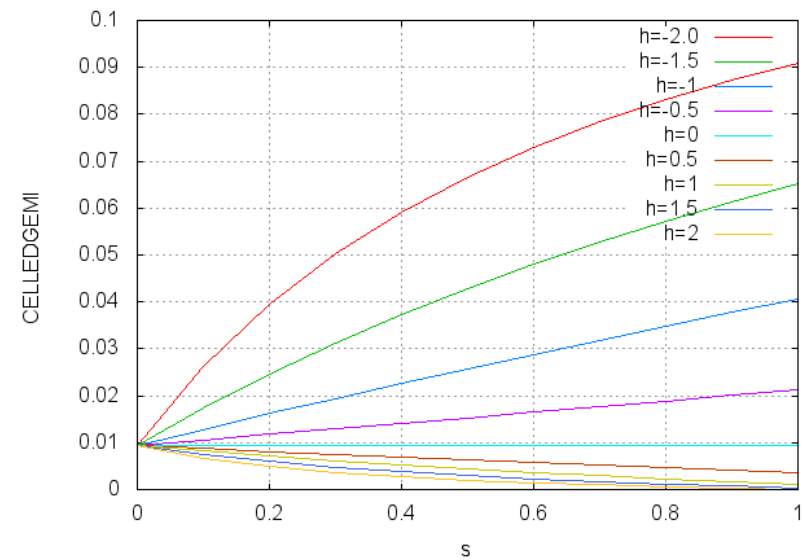

(d) Cell edge performance $\gamma_{C E}=f(h, \underline{\mathbf{s}})$ in $\mathrm{b} / \mathrm{s} / \mathrm{Hz}$

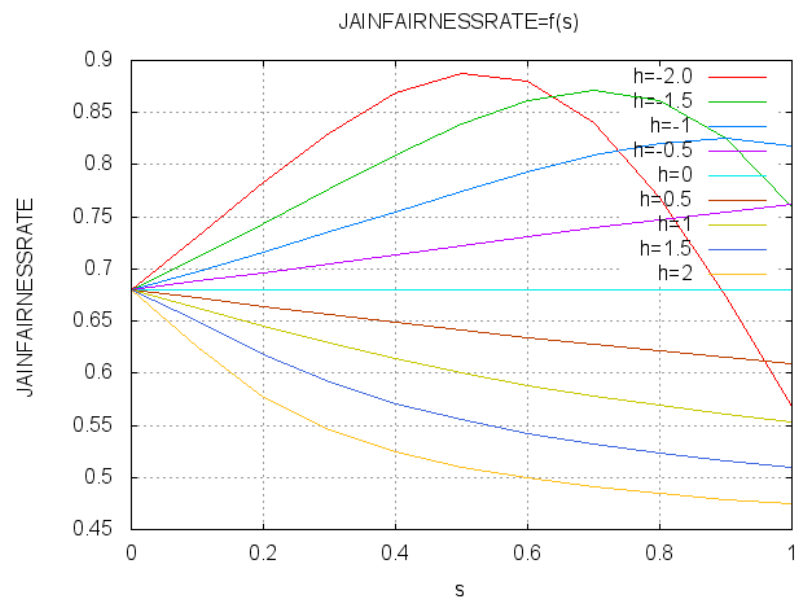

(f) Rate fairness $J_{R}=f(h, \underline{\mathbf{s}})$

Fig. 4. Average and cell edge spectral efficiency results $\left(\bar{\gamma}\right.$ and $\left.\gamma_{C E}\right)$ and rate fairness results $J_{R}$ depending on scheduling parameters $h$ and $s$

[12] R. Gaeta, M. Gribaudo, D. Manini, and M. Sereno, "On the use of Petri nets for the computation of completion time distribution for short TCP transfers," Proceedings of the 24th international conference on Applications and theory of Petri nets, vol. LNCS, pp. 181-200, 2003.

[13] R. Schoenen, "Credit-based flow control for multihop wireless networks and stochastic Petri nets analysis," in Proceedings of the CNSR, Ottawa, May 2011

[14] J. Monserrat, P. Sroka, G. Auer, J. Cabrejas, D. Martin, A. Mihovska R. Rossi, A. Saul, and R. Schoenen, "Advanced radio resource management for IMT-Advanced in WINNER+ (II)," in Proc. ICT-MobileSummit 2010, Florence, Italy, Jun 2010.

[15] R. Schoenen and F. Qin, "A Closed Loop Control Model separating Resource and Packet Scheduling in Multihop Cellular Networks," in Proc. of the European Wireless, Lucca, Italy, Apr 2010.
[16] M. Salem, A. Adinoyi, H. Yanikomeroglu, and D. Falconer, "Opportunities and challenges in OFDMA-based cellular relay networks: A radio resource management perspective," Vehicular Technology, IEEE Transactions on, June 2010.

[17] V. Živojnović, R. Schoenen, and H. Meyr, "On Retiming of Multirate DSP Algorithms," in Proceedings of the IEEE International Conference on Acoustics, Speech and Signal Processing, vol. VI, Atlanta, May 1996.

[18] L. Kleinrock, Queueing Systems, Vol. I: Theory. New York: John Wiley \& Sons, 1975.

[19] ITU, "Report ITU-R M2135-1; Guidelines for evaluation of radio interference technologies for IMT-Adcanced", 2009.

[20] A. B. Sediq, R. Schoenen, H. Yanikomeroglu, G. Senarath, and Z. Chao, "A novel distributed inter-cell interference coordination scheme based on projected subgradient and network flow optimizations," in PIMRC'2011. 


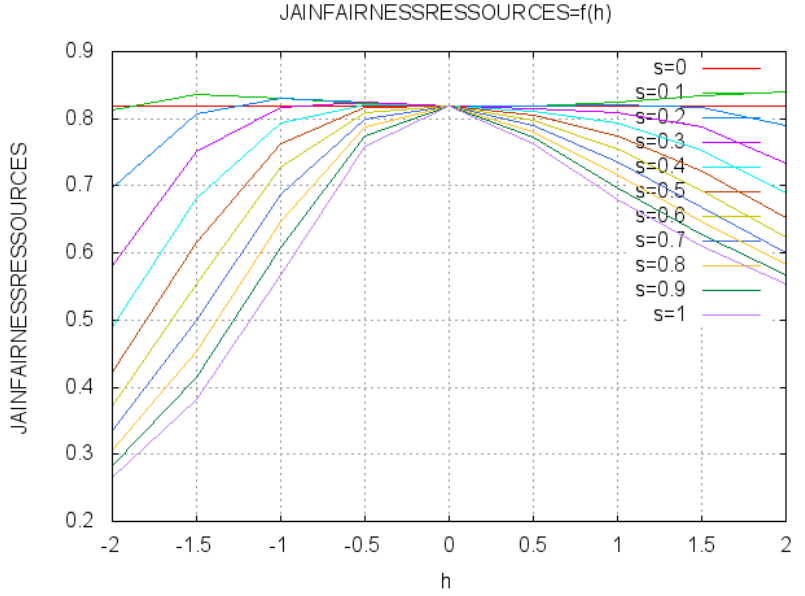

(a) Resource fairness $J_{S}=f(\underline{\mathbf{h}}, s)$

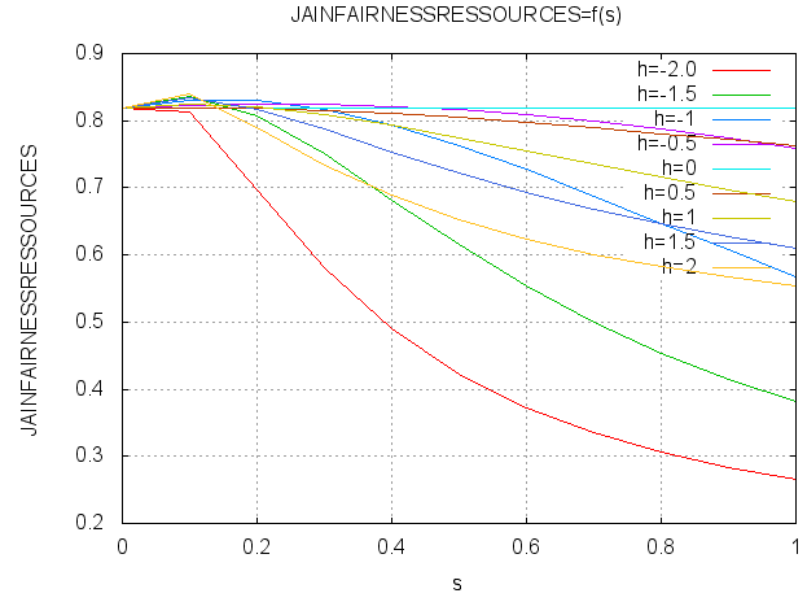

(b) Resource fairness $J_{S}=f(h, \underline{\mathbf{s}})$

Fig. 5. Resource Fairness results $J_{S}$ depending on parameters $h$ and $s$

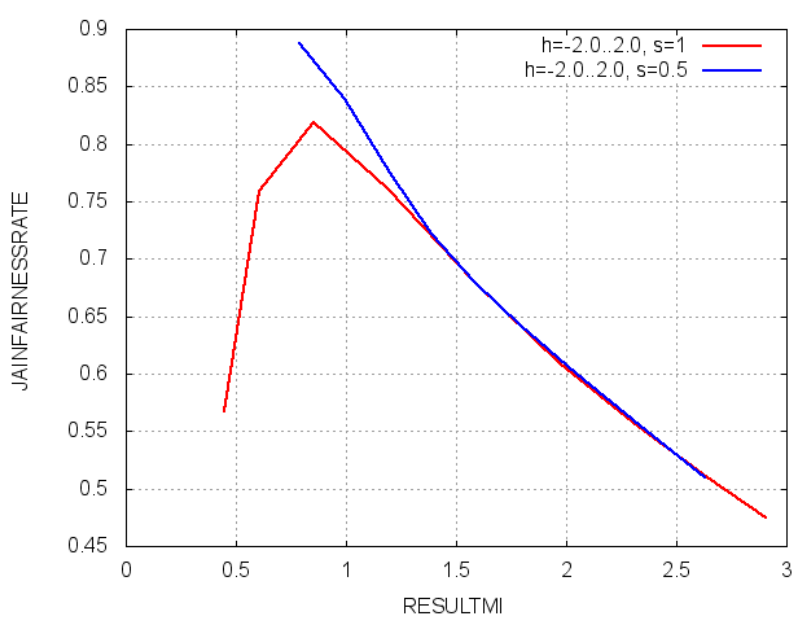

(a) Rate fairness $J_{R}$ versus $\bar{\gamma}$ in $\mathrm{b} / \mathrm{s} / \mathrm{Hz}$

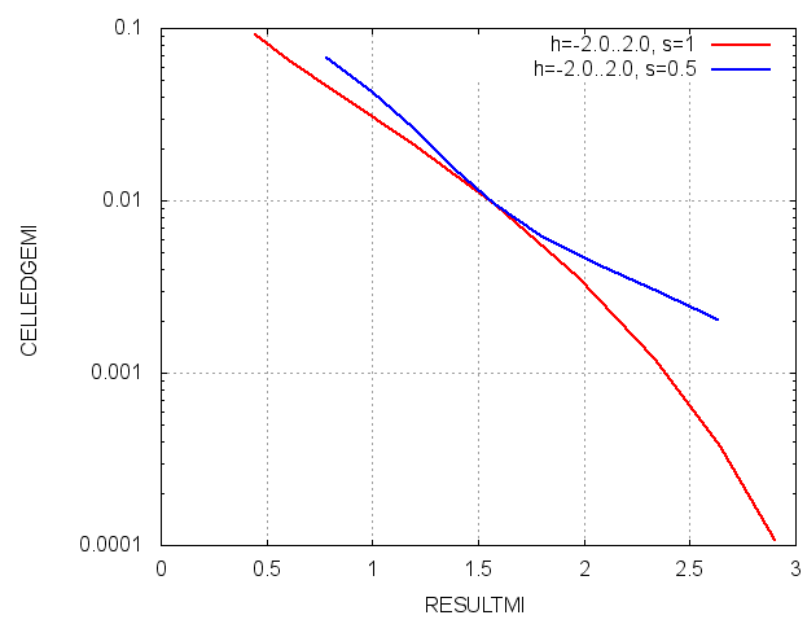

(b) $\gamma_{C E}$ versus $\bar{\gamma}$ (cell edge versus total average) in $\mathrm{b} / \mathrm{s} / \mathrm{Hz}$

Fig. 6. Tradeoff between average spectral efficiency $\bar{\gamma}$ (x-axis, in b/s/Hz) and rate fairness $J_{R}$ or cell edge performance $\gamma_{C E}(\mathrm{~b} / \mathrm{s} / \mathrm{Hz}$ )

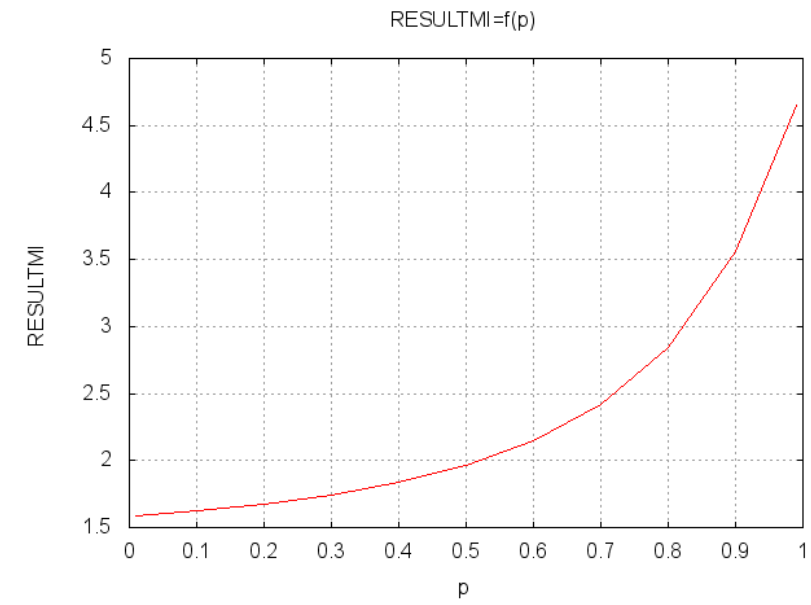

(a) $\bar{\gamma}=f(p)$ in $\mathrm{b} / \mathrm{s} / \mathrm{Hz}$

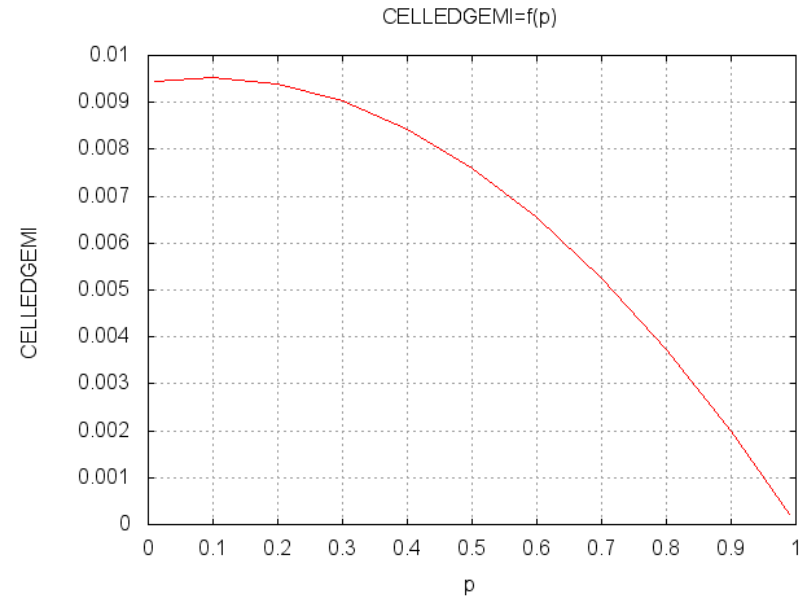

(b) $\gamma_{C E}=f(p)$ in $\mathrm{b} / \mathrm{s} / \mathrm{Hz}$

Fig. 7. SPN analysis results of the UIL scenario. Parameter $p$ is the probability of a user moving to a position of better spectral efficiency 